\title{
Effect of laughter on salivary endocrinological stress marker chromogranin A
}

\author{
Masahiro Toda ${ }^{1}$, Shinsuke Kusakabe ${ }^{1}$, Shingo Nagasama ${ }^{2}$, Kazuyuki Kitamura ${ }^{2}$ and Kanehisa Morimoto ${ }^{1}$ \\ ${ }^{1}$ Department of Social and Environmental Medicine, Osaka University Graduate School of Medicine, Osaka and ${ }^{2}$ Research and Develop- \\ ment, Yanaihara Institute Inc., Shizuoka, Japan
}

(Received 15 January 2007; and accepted 29 January 2007)

\begin{abstract}
We investigated the effect of laughter on salivary endocrinological stress marker chromogranin A (CgA). In saliva samples collected from 11 healthy males before and after watching a comic film or a non-humorous control film, salivary $\mathrm{CgA}$ levels were determined by enzyme-linked immunosorbent assay (ELISA). Samples taken after watching the comic film showed increased levels of $\mathrm{CgA}$. This tendency was more pronounced in individuals with lower initial levels of stress. The control samples showed no significant change in CgA levels. Stress score, subjectively evaluated using a visual analog scale, decreased significantly after watching the comic film. These findings suggest that, in addition to a stress relief effect, laughter can bring about feeling uplifted or fulfilled.
\end{abstract}

As reflected by the saying, "Fortune comes to a merry home", empirical observation since ancient times has associated laughter with positive effects. Recent developments in psychoneuroimmunology have made it possible to scientifically investigate the effects of laughter on health. Several studies have found, after people watched comic films, elevated natural killer cell activity $(1,3,13)$, relief of allergic skin wheal responses in patients with atopic dermatitis (5), and higher pain tolerance (18). Besides the report by Berk et al. (2) on reduced serum cortisol levels after watching a comic film, we know of few studies on the effects of laughter on endocrinological stress markers. In the present study, therefore, we examined how laughter affected the salivary endocrinological stress marker chromogranin $\mathrm{A}(\mathrm{CgA})$. $\mathrm{CgA}$ is an acidic glucoprotein that is released along with catecholamines from the adrenal medulla and the sympathetic nerve endings $(11,12,19)$. A recent

Address correspondence to: Prof. Kanehisa Morimoto Department of Social and Environmental Medicine, Osaka University Graduate School of Medicine, 2-2 Yamada-Oka, Suita, Osaka 565-0871, Japan Tel: +81-6-6879-3920, Fax: +81-6-6879-3929

E-mail: morimoto@envi.med.osaka-u.ac.jp study has reported that $\mathrm{CgA}$ is produced by human submandibular glands and secreted into saliva (9). Salivary $\mathrm{CgA}$ has gained attention as a novel stress marker. Whereas cortisol has long been assayed as a stress marker that reflects both mental and physical stress, levels of salivary CgA correlate only with mental stress $(7,8)$.

After giving written informed consent, 11 healthy male university students aged 19-23 years old were recruited to take part in the study. None were receiving any medication. On different days, they watched a comic film and a non-humorous control film (landscape scenes) for $30 \mathrm{~min}$. The comic film was part of a TV comedy show featuring popular Japanese comedians. Film viewing and sampling followed the same experimental protocol, but the order of showing the films was randomized to cancel possible sequential viewing effects. As Fig. 1 shows, saliva samples were collected immediately before and after watching the film, and $30 \mathrm{~min}$ after the end of viewing. Although the circadian variation of $\mathrm{CgA}$ is still not fully established, in a previous study, we confirmed that salivary $\mathrm{CgA}$ levels were likely to be stable in the afternoon (15). Saliva samples were collected using the Salivette system (Sarstedt Co. Ltd., Nümbrecht, Germany). In this 


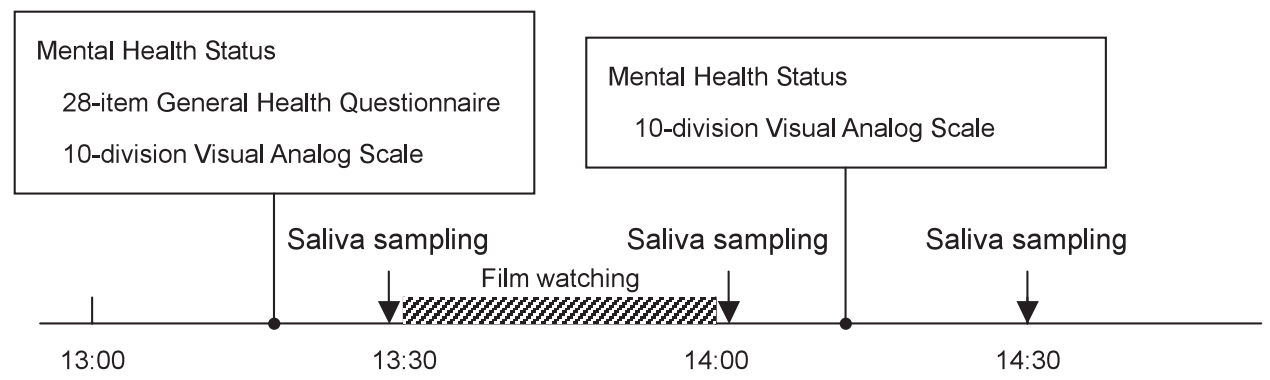

Fig. 1 Study protocol.

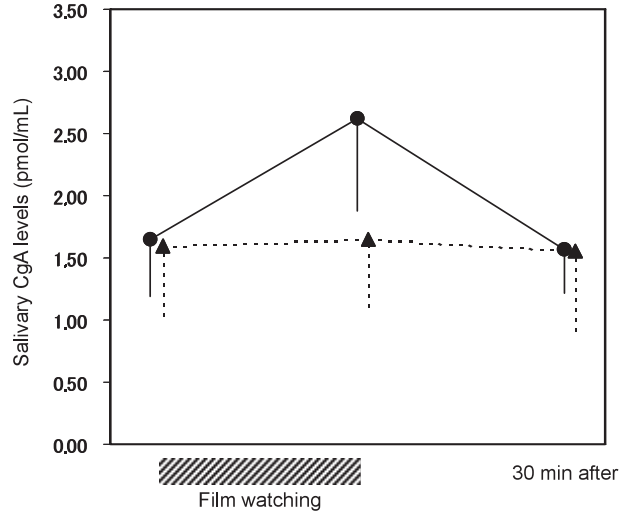

Fig. 2 Changes in mean values $( \pm \mathrm{SE})$ for salivary $\mathrm{CgA}$ (chromogranin A) levels in samples taken during the comic film protocol (solid line, $n=11$ ) and in control samples (dashed line, $n=11$ ).

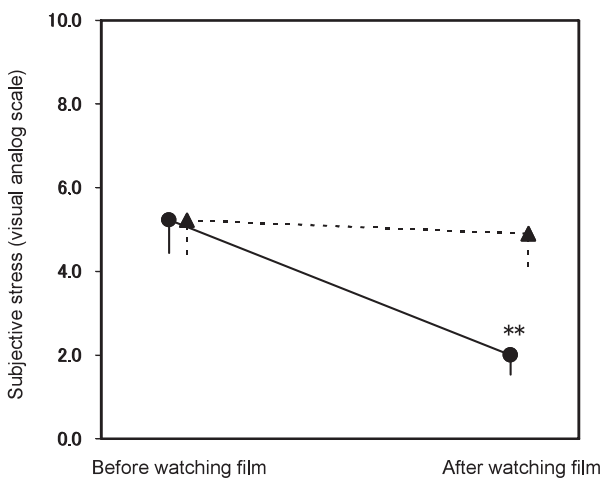

Fig. 4 Changes in subjective stress (mean values $\pm S E$ ) after watching the comic film (solid line, $n=11$ ) and the non-humorous control film (dashed line, $n=11$ ). ${ }^{* *} p<0.01$ (Student's paired t test).

method, saliva samples are extracted from cotton wads that subjects had held in their mouths (for $2 \mathrm{~min}$ ) by centrifuging (at 3,000 rpm for $15 \mathrm{~min}$ ). These samples were stored at $-80^{\circ} \mathrm{C}$ until assay. Using a previously described method (6), levels of salivary $\mathrm{CgA}$ were evaluated using enzyme-linked

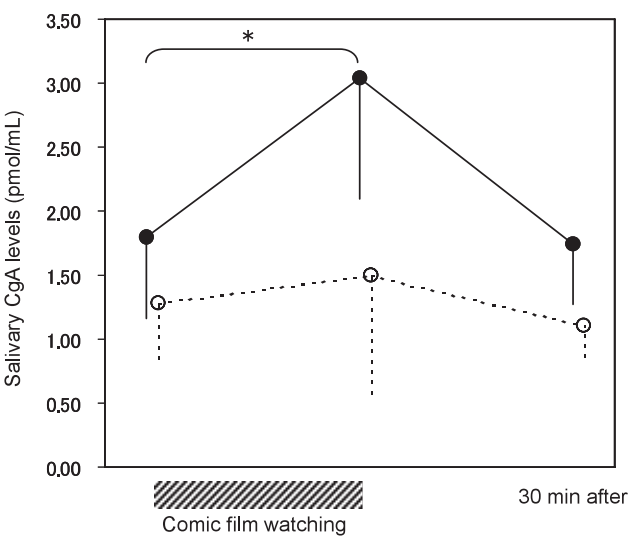

Fig. 3 Changes in mean values $( \pm \mathrm{SE})$ for salivary $\mathrm{CgA}$ (chromogranin A) levels in low GHQ-28 score group (solid line, $n=8$ ) and high GHQ-28 score group (dashed line, $n=3$ ) samples taken during the comic film protocol. ${ }^{*} p<0.05$ (repeated measures ANOVA and Bonferroni's test).

immunosorbent assay (ELISA). Before the experiment, by means of written questionnaire, mental health status was evaluated using GHQ-28 (28-item General Health Questionnaire). Subjects scoring 8 points or more were placed in the high-score group (4). In addition, subjective stress immediately before and after watching the film was measured using a ten-division visual analog scale. ANOVA with repeated measures was performed to detect intergroup and time-related differences. Bonferroni's test was used for multiple comparisons. Values were considered to be significantly different when $p<0.05$.

Fig. 2 shows changes, among all subjects during the sampling period, in the mean levels of salivary $\mathrm{CgA}$. There was no significant difference in salivary CgA levels before and $30 \mathrm{~min}$ after watching either the comic film or the control film. Samples taken immediately after watching the comic film, however, tended to have higher levels of $\mathrm{CgA}$. Fig. 3 shows changes, during the comic film protocol, in the mean levels of $\mathrm{CgA}$ for viewers grouped accord- 
ing to their GHQ-28 scores. The low-score group showed significantly higher CgA levels immediately after watching the film, but samples from the highscore group showed no significant change in the level of CgA. Subjective perception of stress, evaluated using a visual analog scale, decreased significantly after watching the comic film (Fig. 4). Before and after watching the control film, there was no such change.

Salivary CgA levels showed a tendency to increase immediately after watching the comic film (Fig. 2). As mentioned above, levels of salivary $\mathrm{CgA}$ are considered an index of only mental stress $(7,8)$. The concept of mental stress embraces both positive (eu-stress) and negative (di-stress) aspects (10), so perhaps the levels of $\mathrm{CgA}$ after watching the comic film resulted from positive mental eu-stress rather than negative di-stress. This conjecture is supported by significantly lower subjective perceptions of stress after watching the comic film (Fig. 4). Furthermore, in recent studies, we found that moderate stress (eu-stress) such as short leisure trips, spa bathing, or coffee intake significantly increases salivary $\mathrm{CgA}$ levels $(14,16,17)$, suggesting that $\mathrm{CgA}$ may be a good index of eu-stress. When results were analyzed according to GHQ-28 scores, only the low-score group showed a significant increase in CgA levels after watching the comic film (Fig. 3). This finding is also in line with results from our previous studies. Increased $\mathrm{CgA}$ levels during short leisure trips or associated with spa bathing were more pronounced in people reporting low initial levels of stress $(14,16)$. Furthermore, increased $\mathrm{CgA}$ levels after coffee intake were found in healthy women, but were not found in pregnant women, who scored high for stress (17). Thus, persons with positive characteristics related to mental health status are particularly likely to experience beneficial effects, in the form of feeling uplifted or fulfilled, from the evocation of laughter and other eu-stress. The present findings suggest that laughter is likely to relieve stress. In addition to such relief, for people who are not otherwise feeling undue stress, the experience of laughter may result in an uplifted or fulfilled feeling. On the other hand, results for the high-stress group showed no significant change in $\mathrm{CgA}$ levels before and after watching the comic film: this finding may be due to low $(n=3)$ sample size of this group and has to be confirmed by the study of larger populations.

While our findings indicate that laughter probably has beneficial effects, the research does have some limitations. For example, a previous study has sug- gested that laughter is a phenomenon with both experiential and expressive aspects (13). Our protocol did not consider, however, the expressive aspects of laughter, for example, by evaluating facial expressions and physical actions, or recording the various kinds and qualities of laughter. Studies of the relationship between laughter and stress would benefit from a more multifaceted approach.

\section{REFERENCES}

1. Bennett MP, Zeller JM, Rosenberq L and McCann J (2003) The effect of mirthful laughter on stress and natural killer cell activity. Altern Ther Health Med 9, 38-45.

2. Berk LS, Tan SA, Fry WF, Napier BJ, Lee JW, Hubbard RW, Lewis JE and Eby WC (1989) Neuroendocrine and stress hormone changes during mirthful laughter. Am J Med Sci 298, 390-396.

3. Berk LS, Felten DL, Tan SA, Bittman BB and Westengard J (2001) Modulation of neuroimmune parameters during the eustress of humor-associated mirthful laughter. Altern Ther Health Med 7, 62-76.

4. Goldberg DP (1978) Manual of the General Health Questionnaire. NFER Publishing, Windsor.

5. Kimata H (2004) Laughter counteracts enhancement of plasma neurotrophin levels and allergic skin wheal responses by mobile phone-mediated stress. Behav Med 29, 149-152.

6. Nagasawa S, Nishikawa Y, Jun L, Futai Y, Kanno T, Iguchi K, Mochizuki T, Hoshino M, Yanaihara C and Yanaihara N (1998) Simple enzyme immunoassay for the measurement of immunoreactive chromogranin A in human plasma, urine and saliva. Biomed Res 19, 407-410.

7. Nakane H, Asami O, Yamada Y, Harada T, Matsui N, Kanno $\mathrm{T}$ and Yanaihara N (1998) Salivary chromogranin A as an index of psychosomatic stress response. Biomed Res 19, 401406.

8. Nakane H, Asami O, Yamada Y and Ohira H (2002) Effect of negative air ions on computer operation, anxiety, and salivary chromogranin A-like immunoreactivity. Int $J$ Psychophysiol 46, 85-89.

9. Saruta J, Tsukinoki K, Sasaguri K, Ishii H, Yasuda M, Osamura YR, Watanabe Y and Sato S (2005) Expression and localization of chromogranin A gene and protein in human submandibular gland. Cells Tissues Organs 180, 237-244.

10. Selye H (1978) The Stress of Life. McGraw-Hill, New York.

11. Smith WJ and Kirshner N (1967) A specific soluble protein from the catecholamine storage vesicles of bovine adrenal medulla. Mol Pharmacol 3, 52-62.

12. Smith AD and Winkler H (1967) Purification and properties of an acidic protein from chromaffin granules of bovine adrenal medulla. Biochem $J$ 103, 483-492.

13. Takahashi K, Iwase M, Yamashita K, Tatsumoto Y, Ue H, Kuratsune H, Shimizu A and Takeda M (2001) The elevation of natural killer cell activity induced by laughter in a crossover designed study. Int J Mol Med 8, 645-650.

14. Toda M, Makino H, Kobayashi H, Nagasawa S, Kitamura K and Morimoto K (2004) Medical assessment of the health effects of short leisure trips. Arch Environ Health 59, 717-724.

15. Toda M, Morimoto K, Nagasawa S and Kitamura K (2004) Effect of snack eating on sensitive salivary stress markers cortisol and chromogranin A. Environ Health Prev Med 9, 27-29. 
16. Toda M, Morimoto K, Nagasawa S and Kitamura K (2006) Change in salivary physiological stress markers by spa bathing. Biomed Res 27, 11-14.

17. Tsubouchi H, Shimoya K, Hayashi S, Toda M, Morimoto K and Murata Y (2006) Effect of coffee intake on blood flow and maternal stress during the third trimester of pregnancy. Int J Gynecol Obstet 92, 19-22.
18. Weisenberg M, Tepper I and Schwarzwald J (1995) Humor as a cognitive technique for increasing pain tolerance. Pain 63, 207-212.

19. Winkler H and Fischer-Colbrie R (1992) The chromogranins $\mathrm{A}$ and B: the first 25 years and future perspectives. Neuroscience 49, 497-528. 